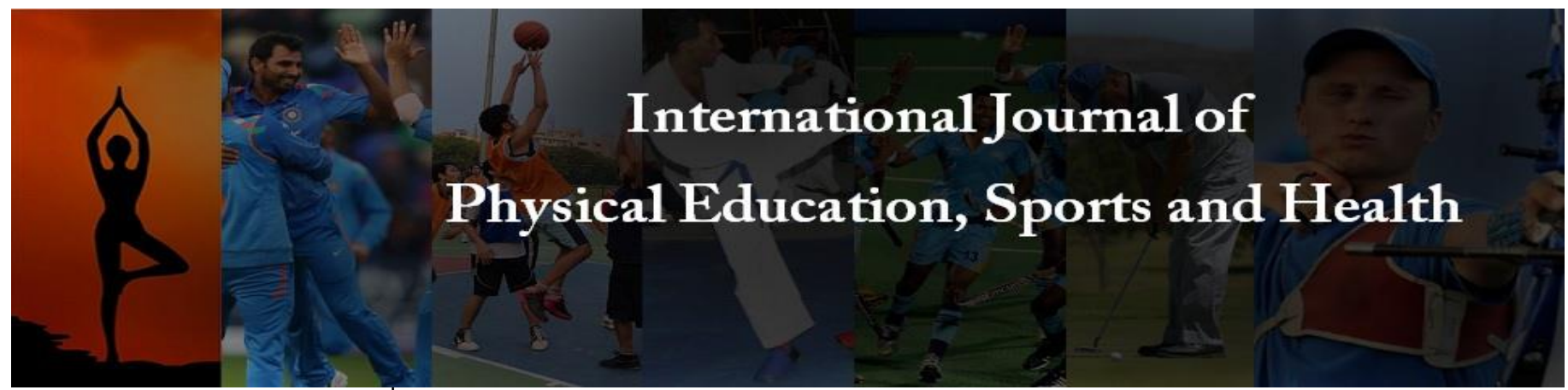

P-ISSN: 2394-1685

E-ISSN: 2394-1693

Impact Factor (ISRA): 5.38

IJPESH 2022; 9(1): 01-04

(C) 2022 IJPESH

www.kheljournal.com

Received: 15-10-2021

Accepted: 02-12-2021

\section{Shaheed Ahmed}

PG in MPT Sports Medicine, Jamia Hamdard, New Delhi, India

\section{Kalpana Zutshi}

Associate Professor, Dept of

Rehabilitation Sciences, SNSHA,

Jamia Hamdard, New Delhi,

India

Shaji John

HOD, Sports Sciences, Manav Rachna University, Faridabad, Haryana, India
Corresponding Author: Shaheed Ahmed

PG in MPT Sports Medicine, Jamia Hamdard, New Delhi, India

\section{Effect of core training on football specific skills speed dribbling}

\author{
Shaheed Ahmed, Kalpana Zutshi and Shaji John
}

\section{Abstract}

Objectives: To evaluate the effect of core training on football-specific skills Speed dribbling.

Methods: The design of the study was a pre- and post-test-controlled trial. This research study was carried out at the Cambridge school and Ashoka football academies in Noida, India. Thirty healthy male footballers were selected for the study. The experimental group underwent core training for four weeks in addition to conventional training, while the control group only did conventional training.

Results: At the end of 4 weeks of training, both groups showed improvement in their skills; however, the improvement in the experimental group was significantly better than in the control group.

Conclusion: Core training improves football-specific Speed dribbling skills hence core training should be added to regular training of players.

Keywords: Core training, football, skills speed dribbling, Noida, India

\section{Introduction}

Football is the most popular sport in the world and is performed by men, women, children and adults ${ }^{[1]}$. With over 40 million registered players and many more hundreds of millions of people purported to play the game. There are restrictions on how the game may be played; the main one is that the ball may be propelled by any part of the body except the arms and hands. Thus varieties of strategies and skills have evolved to enable the objective of scoring goals to be accomplished ${ }^{[2]}$.

Football performance depends upon a myriad of factors such as technical, biomechanical, tactical, mental and physiological areas ${ }^{3}$. One of the reasons that football is so popular is that players do not need to have an extraordinary capacity within any of these performance areas, but possess a reasonable level within all areas ${ }^{1}$. It has been found that the physical capacity of footballers influences their technical performance and tactical choice as well as the frequency of injuries ${ }^{[4]}$.

Core strengthening has been the subject of much research since the early 1980s. Research has highlighted benefits of training for people with back pain and for carrying out everyday activities. Less research has been performed on the benefit of core training for athletes specifically. A further confounding factor is that, because of the different demands on the core musculature during everyday activities (low load, slow movements) and sporting activities (high load, resisted, dynamic movement), research performed in the rehabilitation sector cannot be applied to the sporting environment and subsequently data regarding core training programmes and their effectiveness on sporting performance are lacking ${ }^{[5]}$.

The central core of the body is seen as being pivotal for efficient biomechanics, to function with maximal force generation and to minimize joint loads in all types of activities ranging from throwing to running. It allows optimal production, transfer and control of force and motion to the terminal segment in integrated athletic activities. Core muscle activity is best understood as the preprogrammed integration of local, single joint muscles and multi joint muscles to provide stability and produce motion. This results in proximal stability for distal mobility, a proximal to distal patterning of generation of force, and the creation of interactive moments that move and protect distal joints ${ }^{[6]}$.

So it's clear that core strengthening enhances the coordination of the lower limb which is an essential component of the football-specific skills. 
Technical skills are also important to footballer performance. Factors such as acceleration, running velocity, jumping height and capacity to release energy are of major importance ${ }^{[3]}$.

The most important variables for measuring performance in a sport such as football, are physical conditioning and technical and tactical performance. However because of the complexity of the game of football, it is difficult to ascertain the relative importance of each of these variables ${ }^{[7]}$.

Without the performance enhancement training effects athlete may not be motivated to participate in training, so we are using footballer specific skills to see the effects of core strengthening ${ }^{[8]}$. Footballer specific skills includes eight skills [7]. Skills in football are more complex than indicated by field tests, level of competition and so on, and includes a combination of physical functional behavioral and perceptual features ${ }^{[9]}$.

\section{Aim of the study}

The aim of our study was to investigate the effect of core training on footballer specific skills.

\section{Methodology \\ Design and Sampling}

The design of the study was a pre- and post-test-controlled trial. This research study was carried out at the Cambridge school and Ashoka football academies in Noida, India. Thirty healthy male footballers with mean age were selected for the study. The experimental group underwent core training for four weeks in addition to conventional training, while the control group only did conventional training. Subjects were conveniently selected on the basis of inclusion and exclusion criteria and were then randomly assigned to either the core training or conventional training groups.

We gave core training to core training group and conventional training group received conventional training and measured their performance by soccer specific skills.

\section{Procedure}

Ethical approval/Informed Consent: Their coach and parents were given an informed consent and the consent forms were obtained from the coach and subjects prior to participation.

Testing of the footballer specific skills was as done a day prior to initiation of training (pre-test) and at the completion of training (post-test), after a period of four weeks of training. Prior to each football-specific test, subjects were explained how to do the test and it was demonstrated to them. They were then asked to perform three practice trials juggling tests of football skills provided an assessment profile for each players.

Speed dribbling: This test allows assessment of coordinated dribbling under time pressure and assessment of speed. On the signal ready-go the player starts with the ball from behind the line (1). After 5 meters he dribble to the right, around the first post of a triangle (2). Following the set order, he dribble around the other posts. After 10 meter he dribble around a block (3). Then after 8 meter he plays the ball around one side of a square (4) and runs around the other side (5) to collect it (6). Then he sprints through a gate and puts his foot on the ball. (7). the examiner measures the time taken from the go signal until the player has the ball under his foot. Measurement is made with a stopwatch in units of 0.1 seconds.

\section{Training Program}

Subjects were asked to be regular for the training as deemed by the researcher. Progression of exercises proceeded as follows: Unloaded to loaded, simple to complex, stable to labile, isometric to concentric then eccentric, endurance to strength, slow to fast movements.

Core exercises were organized into different a track to progress the players through increasingly difficult exercises for example Isometric holds with co-contraction were performed first, then, by adding movement of the extremities and resistance, the intensity of the exercises was increased. The number of exercises and repetitions were determined by the player's ability to perform the exercise and if a particular exercise could not be performed appropriately, the progression of exercise was halted and the exercise was made easier.

\section{Warm up}

The warm up consisted of light aerobic work, i.e. jogging for 5 to 10 minutes, followed by stretching exercises. (Static and dynamic of upper and lower limbs that include shoulder, elbow wrist, neck, back, hip, knee \& ankle).

\section{Core Exercises}

The Bridge track incorporated a Ball Bridge, Floor Bridge, a Marching Ball Bridge with single leg, and a Hamstring Bridge with feet on an exercise ball. The Dead bug track included an Isometric ball squeeze, and alternate leg movements with a stable trunk. The Quadruped track included alternate leg movements with stable trunk, and the Supine track, included a Centre crunch, Oblique crunch. The Side Support track consisted of a side support with knee flexed to 90 degrees. The Prone track involved repetitive spine extensions over an exercise ball abducted and lastly, the Standing track included a full ball squat on two legs plus a full ball squat on one leg.

\section{Cool down exercises}

Cool down involved light aerobic work, i.e. jogging for 5 to 10 minutes, and stretching (dynamic) exercises.

\section{Frequency of training}

The subjects performed the core strengthening exercises thrice per week for 4 weeks on alternating days, (Monday, Wednesday and Friday) performing repetition and sets according to the specific exercises. Both groups continued with their conventional training exercises.

\section{Analysis \\ Data analysis was performed using SPSS-15 for window software. Descriptive data was used to compare all groups at base line for age, weight, height, and experience. The Speed dribbling dependent variable were measured at two levels pre- intervention and post-intervention and were analyzed at independent level of dependent variables (between groups) and dependent level of dependent variables (within groups). Level of significance $\mathrm{p} \leq 0.05$.}

\section{Results}

Descriptive statistics were used to analyze subject characteristics. Statistical analysis revealed that there was no significant difference between the experimental group and control group at baseline, which shows that the groups were homogenous. The dependent variable - Speed dribbling were measured at two levels pre intervention and post intervention and were analyzed at independent level of dependent variables (Between groups) and dependent level of dependent variables (Within groups). Within group analysis for all these 
groups was done using paired $\mathrm{T}$ - test. Between groups analysis was done using independent $\mathrm{T}$ test. The significance level was set to $\mathrm{P} \leq 0.05$.

\section{Speed \\ Between groups \\ Pre-intervention}

The Pre-intervention analysis between experimental and control group mean scores of speeds showed significant result with t value 4.595 and $p$ value .000 .

\section{Post-intervention}

The post intervention analysis between experimental and control group mean scores of post speed showed significant result with t value 5.077 and p value .000 .

\section{Within group analysis}

When Experimental group analysed for the pre and post intervention mean scores, results showed significant result with $t$ value 5.03 and $p$ value .000 .

Comparison of Control group analysed for pre and post mean scores results showed significant results with t value 3.816 and $\mathrm{p}$ value .002 .

Table 1: Speed within and between groups comparison

\begin{tabular}{|c|c|c|c|c|}
\hline Speed & Pre & Post & T & P \\
\hline Experimental & $122.89 \pm 3.47$ & $122.16 \pm 3.44$ & 5.036 & .000 \\
\hline Control & $128.04 \pm 2.59$ & $127.59 \pm 2.30$ & 3.816 & .002 \\
\hline T & 4.595 & -5.077 & \multicolumn{2}{|}{} \\
\hline P & .000 & .000 & & \\
\hline
\end{tabular}

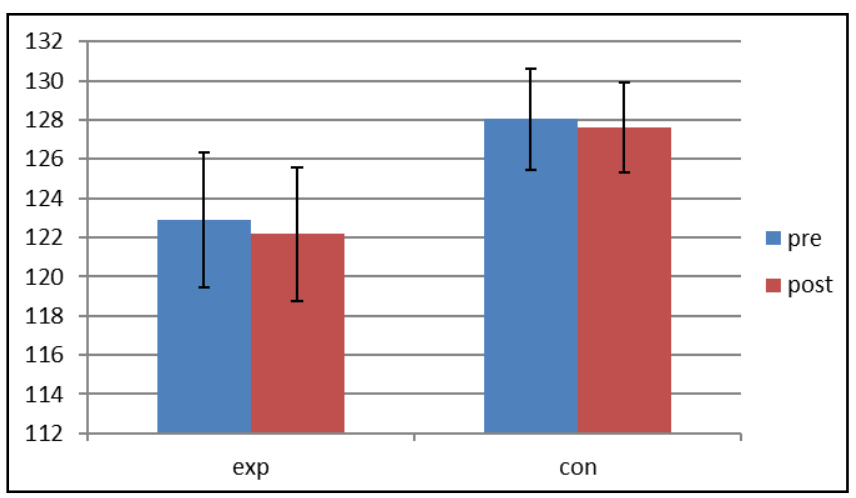

Fig 1: Speed within group comparison

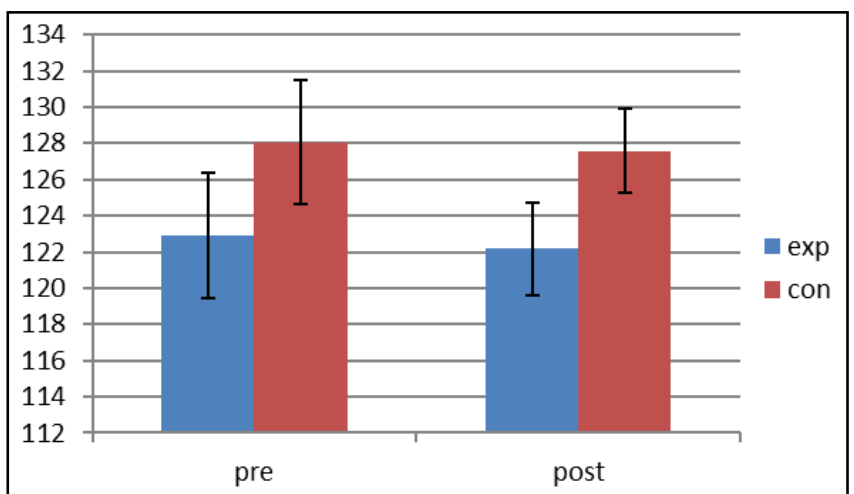

Fig 2: Speed between groups comparison

\section{Discussion}

Core stabilization has become an important component in the athletic arena. Core strengthening has been the subject of research since the early 1980s. Researchers have highlighted the benefits of these training processes on people with back pain and increased capabilities for carrying out everyday activities. However less research has been carried on the benefit of the core training for athletes ${ }^{[5]}$.

The analysis revealed that both groups improved statistically during the study period but the experimental group showed a greater statistical improvement when compared with the control group, which emphasizes the fact that core muscular training improves these specific skills in the footballers. These findings are in accordance with the previous conducted research on core training in which physical performance improved.

Example A study by Myer et al. ${ }^{[8]}$ was conducted to examine the effect of 6-week program of neuromuscular training on a single leg hop and hold, distance test. Distance jumped and held increased following the core training program. He found stability and strength improvements and enhanced performance following program.

Vezina ${ }^{[10]}$ also studied the effects of core training on 24 healthy men on stability and EMG was recorded from muscle sites. Exercise were divided into 2 phases; movement and stabilization concluded that following core training all muscle sites were activated, forming the basis of a stabilizing exercise approach.

Previous studies have largely focused upon various parameters such as strength, stability and balance. Our study has focused more towards the measurement of football specific skills which ultimately helps in determining the overall on-field performance.

There are many tests to measure performance after core training but we have focused mainly on specific skills involved in a game of football, mainly because of: without performance enhancing training effects, (on football specific skills in this study) athletes may not be motivated to participate in training programs ${ }^{[8]}$.

Physiological muscle activation results in several biomechanical effects that allow efficient local and distal function. Pre-programmed muscle activation results in anticipatory postural adjustments (APAs), which position the body to withstand the perturbation to balance created by the forces of kicking, throwing or running. The APAs create the proximal stability for distal mobility ${ }^{[6]}$. The muscle activation also creates the interactive moments that develop and control forces and loads at joints. Interactive moments are the moments at joints that are created by motion and position of adjacent segments.

They are developed in the central body segment and are key to developing proper force at distal joints and for creating relative bony positions that minimize internal loads at the joint. Interactive moments either provide maximal force at the distal end or they provide precision and stability to the distal end Kibler Ben.

As a result of the activation and interactive and moments, there is a proximal to distal development of force and motion, according to the summation of speed principle that include core activation ${ }^{[6]}$.

W. Ben Kibler ${ }^{[6]}$ core stability creates several advantages for integration of proximal and distal segment in generating and controlling forces to maximize athletic function. The larger bulkier muscles in the central core create a rigid cylinder and large moments of inertia against body perturbation while still allowing a stable base for a distal mobility. In addition it, places most of the engine of force development in the central core, allowing small changes in rotation around the central core to effect large change in rotation in the distal segments. Because of the need for relatively smaller mass in the 
peripheral segments, the moments of inertia in these distal areas is less, allowing the summation of higher velocities.

Finally by allowing joint force control to be largely influenced and controlled by pre-programmed muscle activation patterns and interactive moments develop through the core activation. Instead of being based on local ligament size or feedback based local muscle activation, the ligament can be smaller in size, and the smaller local muscle can be activated for precision and control of performance variables.

Between group analysis of control and experimental has shown that there is improvement in control group also, as various components of core training were already a part of regular training program but significant differences in the improvement level between experimental and control group suggests that specifically targeting the core would help in further enhancing the skills involved in the game of football as has been shown by experimental group.

There was significant improvement in some skills. It may be because of more use of these skills in practice and in matches. Skills which have shown improvement are performed by almost all players of the team as a part of conventional training programs irrespective of the position of the players in the match or in practice.

\section{Significance of study}

Thus the significance of our study is to emphasize the fact that a specific core exercises enhances the footballer specific skills along with conventional training as compared to conventional training alone.

\section{Limitation of the study}

Psychological factors mainly player's motivation levels were not considered.

There was lack of control over external factors like temperature, diurnal variation in training.

\section{Suggestion and scope for future research}

Sports specific performance tests may also be assessed in other sports related research.

The study can be carried out with large number of players, and with female players to get more clarity about role of core training.

\section{Conclusion}

There is significant increase in footballer specific juggling skills in core training group in addition to the conventional training. Thus core training should be incorporated in preseason and in season training program to enhance football specific skills.

\section{References}

1. Temas Stolen. Physiology of soccer,Sports medicine 2005;35(6):501-536.

2. Lees Adriae. Biomechanics of soccer: A review Journal of sports medicine 1998;16:211-234.

3. Hoff Jan. Training and testing capacities for elite soccer players. Journal of sports sciences 2005;23(6):573-582.

4. Chamari K. Infield and labortary testing in young elite soccer players. British Journal of Sports Medicine 2004;38:191-196.

5. Hibbs E Angela. Optimizing performance by improving core stability and core strength. Sports medicine 2008;38(12):995-1008.

6. Kibler Ben W. The role of core stability in athletic function. Sports medicine 2006;36(3):189-198.
7. Rosch Dieter. Assessment and evaluation of football performance. American journal of sports medicine 2000;28(5):s29-s39.

8. Myer D Gregory. Neuromuscular training improves performance and lower extremity biomechanics in female athlete. Journal of strength and conditioning 2005;19(1):51-60.

9. Malina M Robert. Characteristics of youth soccer player aged 13-15 years classified by skill level. British journal of sports medicine 2007;41:290-295

10. Vezina MJ, et al. muscle activation in therapeutic exercise to improve trunk stability, arch phys med rehabil 2000;81(10);1370-9. 